\title{
The Action of the Steenrod Algebra on the Cohomology of $p$-Compact Groups
}

\author{
By \\ Yutaka Hemmi, Hirokazu NishinoBU ${ }^{* *}$ and Kuniyuki OGI***
}

\begin{abstract}
In the present paper, we determine the action of the Steenrod operations on the cohomology of simply connected $p$-compact groups with no $p$-torsion in the integral homologies for an odd prime $p$. To do so we study simple simply connected $p$-compact groups since any simply connected $p$-compact group with no $p$-torsion in the integral homology is a product of such $p$-compact groups.
\end{abstract}

\section{$\S 1$. Introduction}

A $p$-compact group is a space $X$ with finite mod $p$ cohomology $H^{*}\left(X ; \mathbb{F}_{p}\right)$ together with a homotopy equivalence $X \rightarrow \Omega(B X)$ to the loop space of a path connected $p$-complete space $B X$ which is called the classifying space of $X$ ([9]). In the present paper, we determine the action of the Steenrod operations on the cohomology of simply connected $p$-torsion free $p$-compact groups for an odd prime $p$. Here, by " $p$-torsion free" we mean "to have no $p$-torsion in the integral homology".

Typical examples of $p$-compact groups are the $p$-completion of connected compact Lie groups. For simply connected compact Lie groups, it is well known

\footnotetext{
Communicated by S. Mukai. Received November 27, 2007. Revised May 7, 2008.

2000 Mathematics Subject Classification(s): Primary 55S05; Secondary 57T25, 55P35, $55 \mathrm{P} 60$.

Key words: $p$-compact group, cohomology operation, simple $p$-compact group.

*Department of Mathematics, Faculty of Science, Kochi University, Kochi 780-8520, Japan.

e-mail: hemmi@math.kochi-u.ac.jp

** Department of Mathematics, Faculty of Science, Kochi University, Kochi 780-8520, Japan.

e-mail: nishinobu@math.kochi-u.ac.jp

*** Anritsu Engineering Co., Ltd., 5-1-1 Onna, Atsugi-City, Kanagawa 243-0032, Japan.

e-mail: Kuniyuki.Ogi@anritsu.com
}

(c) 2008 Research Institute for Mathematical Sciences, Kyoto University. All rights reserved. 
how the Steenrod operations act on their cohomologies. In particular, if $X$ is one of the $p$-completion of the Lie groups $S U(n), \operatorname{Spin}(2 n+1), S p(n)$, and $p$-torsion free exceptional Lie groups for an odd prime $p$, then the following property holds:

(P) $X$ has primitive $\bmod p$ cohomology generators $x_{i}(1 \leq i \leq k)$ with

$$
H^{*}\left(X ; \mathbb{F}_{p}\right) \cong \Lambda\left(x_{1}, \ldots, x_{k}\right), \quad \operatorname{deg} x_{i}=2 n_{i}-1\left(n_{1} \leq \cdots \leq n_{k}\right)
$$

such that if $n_{j}-n_{i}=t(p-1)$ with $t>0$, then

$$
\mathcal{P}^{t} x_{i}=\left(\begin{array}{c}
n_{i}-1 \\
t
\end{array}\right) x_{j}
$$

On the other hand, $(\mathcal{P})$ is not necessarily true for $X=\operatorname{Spin}(2 n)_{p}^{\wedge}$, where $Y_{p}^{\wedge}$ denotes the $p$-completion of a space $Y$. However, $\operatorname{Spin}(2 n)_{p}^{\wedge}$ splits as

$$
\operatorname{Spin}(2 n)_{p}^{\wedge} \simeq \operatorname{Spin}(2 n-1)_{p}^{\wedge} \times\left(S^{2 n-1}\right)_{p}^{\wedge},
$$

and $(\mathcal{P})$ holds for $\operatorname{Spin}(2 n-1)_{p}^{\wedge}$ and for an $H$-space $\left(S^{2 n-1}\right)_{p}^{\wedge}$. Thus, we can say that $\operatorname{Spin}(2 n)_{p}^{\wedge}$ splits as a product of $H$-spaces satisfying $(\mathcal{P})$.

Our first purpose is to show that the similar result holds for simple simply connected $p$-torsion free $p$-compact groups for any odd prime $p$. It is proved that if $p$ is an odd prime, then a simply connected $p$-compact group $X$ is simple if and only if its classifying space $B X$ realizes a $p$-adic pseudoreflection group in the list given by Clark and Ewing ([7], [8]). We will show that $(\mathcal{P})$ holds for almost all simple simply connected $p$-torsion free $p$-compact groups. Our proof uses the Clark-Ewing list. Only the exceptions are the loop spaces of generalized Grassmanians.

The generalized Grassmanian $B X(m, q ; n)$ is a $p$-complete space defined for any positive integers $m, q$ and $n$ with $q|m, m| p-1, m>1$ and $n>1$. The space $B X(m, q ; n)$, which realizes the $p$-adic pseudoreflecton group of No. $2 \mathrm{a}$ in the Clark-Ewing list, satisfies

$$
\begin{gathered}
H^{*}\left(B X(m, q ; n) ; \mathbb{F}_{p}\right) \cong \mathbb{F}_{p}\left[u_{1}, \ldots, u_{n-1}, v\right] \\
\operatorname{deg} u_{i}=2 i m, \quad \operatorname{deg} v=2 n q
\end{gathered}
$$

Then the $p$-compact group $X(m, q ; n)=\Omega B X(m, q ; n)$ is a simple simply connected $p$-compact group with

$$
\begin{gathered}
H^{*}\left(X(m, q ; n) ; \mathbb{F}_{p}\right) \cong \Lambda\left(x_{1}, \ldots, x_{n-1}, y\right) \\
\operatorname{deg} x_{i}=2 i m-1, \quad \operatorname{deg} y=2 n q-1 .
\end{gathered}
$$


Note that

$$
\operatorname{Spin}(2 n)_{p}^{\wedge} \simeq X(2,1 ; n) \text { and } \operatorname{Spin}(2 n+1)_{p}^{\wedge} \simeq X(2,2 ; n)
$$

Then, we show the following ${ }^{1}$

Theorem 1.1. If $p$ is an odd prime, then any simple simply connected p-torsion free $p$-compact group other than $X(m, q ; n)$ with $q<m$ satisfies $(\mathcal{P})$.

A complete list for the Steenrod action on the cohomology of simple simply connected $p$-torsion free $p$-compact group is given in section 4 . We note that $\mathcal{P}^{i}$ vanishes for $i \geq p$ except for the ones of Nos. 1 and 2 a for dimensional reasons.

Now it is proved by Adams and Wilkerson [1, Corollary 1.3] and Dwyer, Miller and Wilkerson [8, Theorem 1.1] that if $p$ is an odd prime, then any simply connected $p$-torsion free $p$-compact group splits as a product of simple $p$-compact groups. Thus, from the above theorem we have the following

Theorem 1.2. $\quad$ Let $X$ be a simply connected $p$-torsion free $p$-compact group for an odd prime $p$. Then $X$ splits as

$$
X \simeq X_{1} \times \cdots \times X_{m}
$$

where each $X_{i}$ is a p-compact group satisfying $(\mathcal{P})$ or the $p$-completion of an odd sphere.

For the case of $p=2$, Thomas [21] proved a similar fact to $(\mathcal{P})$ for 2-torsion free simply connected $H$-spaces with primitively generated mod 2 cohomology. Moreover, his result includes a stronger consequence. In fact, he showed that if $x$ is a primitive mod 2 cohomology class with $\operatorname{deg} x=2 n-1$, then for any $t>0$ with $\left(\begin{array}{c}n-t-1 \\ t\end{array}\right) \not \equiv 0 \bmod 2$, there exists a primitive class $y$ with $\operatorname{deg} y=2(n-t)-1$ such that $S q^{2 t}(y)=x$, which is not generally true if $p$ is an odd prime.

Since the above theorem determines the action of the Steenrod algebra on the cohomology of all the simple simply connected $p$-compact groups, we shall have many applications. For example, we have the following

Corollary 1.3. Let $X$ be a simply connected $p$-compact group. Suppose that the action of the Steenrod algebra on $H^{*}\left(X ; \mathbb{F}_{p}\right)$ is trivial. If $p \geq 5$, then $X$ is $p$-regular, that is, $X$ is p-equivalent to a product of odd spheres; if $p=3$, then $X$ is 3-equivalent to a product of odd spheres and some copies of the exceptional Lie group $G_{2}$.

\footnotetext{
${ }^{1}$ After the final submission, the authors found that Theorem 1.1. was independently proved by preprint; D. Davis; Homotopy type and $v_{1}$-periodic homotopy groups of $p$-compact groups; preprint
} 
In the above corollary we need not to assume that $X$ is $p$-torsion free.

The authors would like to thank Yusuke Kawamoto for his useful suggestions.

\section{§2. Simple $p$-Compact Groups}

From now on, we assume that $p$ is an odd prime. Moreover, any $p$-compact group is assumed to be simply connected and $p$-torsion free.

Let $B$ be a $p$-complete space such that the $\bmod p$ cohomology $H^{*}\left(B ; \mathbb{F}_{p}\right)$ is a finitely generated polynomial algebra:

$$
H^{*}\left(B ; \mathbb{F}_{p}\right) \cong \mathbb{F}_{p}\left[u_{1}, \ldots, u_{k}\right], \quad \operatorname{deg} u_{i}=2 n_{i} .
$$

Then, by Dwyer, Miller and Wilkerson [8, Theorem 1.1], there exists a $p$-adic pseudoreflection group $W \subset G L\left(k, \mathbb{Z}_{p}^{\wedge}\right)$ and a map $f: B T \rightarrow B$ equivariant up to homotopy with respect to the natural action of $W$ on $B T$ and the trivial action of $W$ on $B$ such that $f$ induces an isomorphism $H^{*}\left(B ; \mathbb{F}_{p}\right) \cong$ $H^{*}\left(B T ; \mathbb{F}_{p}\right)^{W}$, where $\mathbb{Z}_{p}^{\wedge}$ is the ring of $p$-adic integers, and $B T$ is the classifying space of the $p$-completion of the $k$-dimensional torus $T^{k}$. The space $B$ is called a realization of $W$. We notice that the order of $W$ equals to the product $n_{1} \ldots n_{k}$. If the order of $W$ is divisible by $p$, then $W$ and $\mathbb{F}_{p}\left[u_{1}, \ldots, u_{k}\right]$ are called modular.

All irreducible $p$-adic pseudoreflection groups are classified by Clark and Ewing [7]. Their result is based on the classification of the irreducible complex reflection groups by Shephard and Todd [20]. A $p$-compact group $X$ is simple if its classifying space $B X$ realizes one of the irreducible $p$-adic pseudoreflection groups in the Clark-Ewing list.

Then Theorem 1.2 and Corollary 1.3 are proved from Theorem 1.1.

Proof of Theorem 1.2 from Theorem 1.1. Let $X$ be a $p$-compact group. According to Adams and Wilkerson [1, Corollary 1.3] and Dwyer, Miller and Wilkerson [8, Theorem 1.1], $X$ splits as the product of some simple $p$-compact groups. Moreover, it is proved by [5, Corollary 1.4] that if $q<m$, then

$$
X(m, q ; n) \simeq_{p} X(m, m ; n-1) \times\left(S^{2 n q-1}\right)_{p}^{\wedge} .
$$

Thus, $X$ splits as the product of simple $p$-compact groups other than $X(m, q ; n)$ with $q<m$ and the $p$-completion of odd spheres. Thus we have the result by Theorem 1.1.

Proof of Corollary 1.3. First we note that the integral homology of $X$ has no $p$-torsion. In fact, if it does, then the action of the Bockstein operation 
on $H^{*}\left(X ; \mathbb{F}_{p}\right)$ is non trivial $([13])$. Thus, we have only to show the statement for the case that $X$ is simple by [1, Corollary 1.3] and [8, Theorem 1.1]. Then, by using Theorem 1.1, we see easily that $X$ is either of No. $2 \mathrm{~b}$ of type $(2,6)$ for $p=3$ or of type $\left(n_{1}, \ldots, n_{k}\right)$ with $n_{k}-n_{1}<p-1$. For the former case $X$ is 3 -equivalent to $G_{2}$, while for the later case $X$ is $p$-regular by [12].

\section{§3. Proof of the Main Theorem}

Let $X$ be a $p$-compact group. Then the mod $p$ cohomology of $X$ is an exterior algebra:

$$
H^{*}\left(X ; \mathbb{F}_{p}\right) \cong \Lambda\left(x_{1}, \ldots, x_{k}\right)
$$

for some primitive classes $x_{i}$. The sequence $\left(n_{1}, \ldots, n_{k}\right)$, where $\operatorname{deg} x_{i}=2 n_{i}-1$, is called the type of $X$. We note that the type of $X$ is the same as the one of the polynomial algebra $H^{*}\left(B X ; \mathbb{F}_{p}\right)$, where the type of a polynomial algebra $\mathbb{F}_{p}\left[u_{1}, \ldots u_{k}\right]$ is a sequence $\left(n_{1}, \ldots, n_{k}\right)$ with $\operatorname{deg} u_{i}=2 n_{i}(1 \leq i \leq k)$.

Now we show the following

Lemma 3.1. $\quad$ Let $X$ be a p-compact group of type $\left(n_{1}, \ldots, n_{k}\right)$. Let $n$ be a positive integer with $n \not \equiv 0 \bmod p$ and $n>p$. Suppose that $\mathcal{P}^{1}: P H^{2 t n-2(p-1)-1}$ $\left(X ; \mathbb{F}_{p}\right) \rightarrow P H^{2 t n-1}\left(X ; \mathbb{F}_{p}\right)$ is a monomorphism for $2 \leq t \leq p$, where $P H^{*}(X ;$ $\left.\mathbb{F}_{p}\right)$ is the primitive module of $H^{*}\left(X ; \mathbb{F}_{p}\right)$. Then $\mathcal{P}^{1}: P H^{2 n-2(p-1)-1}\left(X ; \mathbb{F}_{p}\right) \rightarrow$ $P H^{2 n-1}\left(X ; \mathbb{F}_{p}\right)$ is an epimorphism.

Proof. By the assumption, we have $H^{*}\left(B X ; \mathbb{F}_{p}\right) \cong \mathbb{F}_{p}\left[u_{1}, \ldots, u_{k}\right]$ for some $u_{i}(1 \leq i \leq k)$ with $\operatorname{deg} u_{i}=2 n_{i}$. Here, by the same reason as [11, Lemma 4.2] we can choose $u_{i}$ to satisfy the following condition:

If $\mathcal{P}^{1}\left(u_{i}\right)$ is not decomposable then $\mathcal{P}^{1}\left(u_{i}\right)=u_{j}$ for some $u_{j}$; if $\mathcal{P}^{1}\left(u_{i}\right)=$ $\mathcal{P}^{1}\left(u_{j}\right)=u_{k}$ then $u_{i}=u_{j}$.

Then, the assumption that $\mathcal{P}^{1}: P H^{2 t n-2(p-1)-1}\left(X ; \mathbb{F}_{p}\right) \rightarrow P H^{2 t n-1}\left(X ; \mathbb{F}_{p}\right)$ is a monomorphism is equivalent to say that if $\operatorname{deg} u_{s}=2 t n-2(p-1)$ then $\mathcal{P}^{1} u_{s}=u_{m}$ for some $m$. Then we show that if $\operatorname{deg} u_{j}=2 n$ then $\mathcal{P}^{1}\left(u_{i}\right)=u_{j}$ for some $u_{i}$, which implies the result clearly.

Let $J$ be the ideal of $H^{*}\left(B X ; \mathbb{F}_{p}\right)$ generated by all the generators $u_{s}$ with $s \neq j$ so that $H^{*}\left(B X ; \mathbb{F}_{p}\right) / J \cong \mathbb{F}_{p}\left[u_{j}\right]$. Since $n \not \equiv 0 \bmod p$, we have

$$
u_{j}^{p}=\mathcal{P}^{n} u_{j}=\mathcal{P}^{1}\left(n^{-1} \mathcal{P}^{n-1} u_{j}\right) .
$$

Thus we have $\mathcal{P}^{1}\left(H^{*}\left(B X ; \mathbb{F}_{p}\right)\right) \notin J$, and so there is a generator $u_{i}$ with $\mathcal{P}^{1} u_{i} \notin$ $J$. For dimensional reasons, we have $\operatorname{deg} u_{i}=2 t n-2(p-1)$ for some $t$ with 
$1 \leq t \leq p$. Then, by the assumption, $t$ must be 1 and $\mathcal{P}^{1} u_{i}=u_{j}$ since $\mathcal{P}^{1} u_{i}$ is not decomposable.

Next we consider some special cases.

Proposition 3.2. Let $X$ be a p-compact group of type $\left(n_{1}, \ldots, n_{p-1}\right)$ with

$$
n_{i}= \begin{cases}i(p-1), & 1 \leq i \leq p-2 \\ p(p-1), & i=p-1\end{cases}
$$

Then, $X$ satisfies $(\mathcal{P})$.

Proof. We show that we can choose primitive generators $x_{i}(1 \leq i \leq p-1)$ of $H^{*}\left(X ; \mathbb{F}_{p}\right)$ such that

$$
x_{i}=\left(\begin{array}{c}
p-2 \\
i-1
\end{array}\right)^{-1} \mathcal{P}^{i-1} x_{1} \quad \text { for } 2 \leq i \leq p-2 .
$$

Then $(\mathcal{P})$ is satisfied. In fact, if $1 \leq i<j \leq p-2$, then $n_{j}-n_{i}=(j-i)(p-1)$, and we have

$$
\begin{aligned}
\mathcal{P}^{j-i} x_{i} & =\left(\begin{array}{c}
p-2 \\
i-1
\end{array}\right)^{-1} \mathcal{P}^{j-i} \mathfrak{P}^{i-1} x_{1} \\
& =\left(\begin{array}{c}
p-2 \\
i-1
\end{array}\right)^{-1}\left(\begin{array}{c}
j-1 \\
i-1
\end{array}\right) \mathcal{P}^{j-1} x_{1} \\
& =\left(\begin{array}{c}
p-i-1 \\
j-i
\end{array}\right)\left(\begin{array}{c}
p-2 \\
j-1
\end{array}\right)^{-1} \mathcal{P}^{j-1} x_{1} \\
& =\left(\begin{array}{c}
n_{i}-1 \\
j-i
\end{array}\right) x_{j}
\end{aligned}
$$

since $n_{i}-1=(i-1) p+(p-i-1)$ and $j-i<p$. On the other hand, $n_{p-1}-n_{i}=(p-i)(p-1)$ for $i \leq p-2$, and

$$
\mathcal{P}^{p-i} x_{i}=(p-i)^{-1} \mathcal{P}^{1} \mathcal{P}^{p-i-1} x_{i}=0=\left(\begin{array}{c}
n_{i}-1 \\
p-i
\end{array}\right) x_{p-1} .
$$

Now we show (3.2). By the assumption we have

$$
H^{*}\left(B X ; \mathbb{F}_{p}\right) \cong \mathbb{F}_{p}\left[u_{1}, u_{2}, \ldots, u_{p-1}\right]
$$

with

$$
\operatorname{deg} u_{i}= \begin{cases}2 i(p-1), & 1 \leq i \leq p-2 \\ 2 p(p-1), & i=p-1\end{cases}
$$


First, since $\mathcal{P}^{i-1} u_{p-i} \in D^{2} H^{*}\left(B X ; \mathbb{F}_{p}\right)$ for dimensional reasons, we have

$$
\mathcal{P}^{i} u_{p-i}=i^{-1} \mathcal{P}^{1} \mathcal{P}^{i-1} u_{p-i} \in D^{2} H^{*}\left(B X ; \mathbb{F}_{p}\right),
$$

where $D^{t} H^{*}$ is the module of $t$-fold decomposables of an algebra $H^{*}$.

Let $J$ be an ideal of $H^{*}\left(B X ; \mathbb{F}_{p}\right)$ generated by $u_{1}, \ldots, u_{p-2}$. Then, we show that

$$
\mathcal{P}^{p} u_{p-1} \notin J \text {. }
$$

In fact, if $\mathcal{P}^{p} u_{p-1} \in J$, then for dimensional reasons, $\mathcal{P}^{p} u_{i} \in J$ for $1 \leq i \leq p-1$. Thus

$$
\mathcal{P}^{1}\left(H^{*}\left(B X ; \mathbb{F}_{p}\right)\right) \subset J \quad \text { and } \quad \mathcal{P}^{p}\left(H^{*}\left(B X ; \mathbb{F}_{p}\right)\right) \subset J
$$

Then

$$
u_{p-1}^{p}=\mathcal{P}^{p(p-1)} u_{p-1}=-\mathcal{P}^{1} \mathcal{P}^{p(p-1)-2} \mathcal{P}^{1} u_{p-1}-\mathcal{P}^{p} \mathcal{P}^{p(p-1)} u_{p-1} \in J,
$$

and we have a contradiction.

Now we consider the $B P$ cohomology of $B X$ at the prime $p$ (cf. $[10$, Lemma $8.1])$ :

$$
B P^{*}(B X) \cong B P^{*}\left[\left[U_{1}, \ldots, U_{p-1}\right]\right],
$$

where $T\left(U_{i}\right)=u_{i}$ for the natural map $T: B P^{*}(B X) \rightarrow H^{*}\left(B X ; \mathbb{F}_{p}\right)$. We notice that the kernel of $T$ is the ideal $\left(p, v_{1}, v_{2}, \ldots\right)$ generated by $p, v_{1}, v_{2}, \ldots$

Let $r_{t}$ be the Landweber-Novikov operation so that

$$
\chi\left(\mathcal{P}^{t}\right)(T(z))=T\left(r_{t}(z)\right)
$$

for any $z \in B P^{*}(B X)$, where $\chi$ is the canonical anti-automorphism on the mod $p$ Steenrod algebra. In particular, since $\chi\left(\mathcal{P}^{t}\right)=(-1)^{t} \mathcal{P}^{t}$ for $t \leq p$ we have

$$
(-1)^{t} \mathcal{P}^{t}(T(z))=T\left(r_{t}(z)\right) \text { for } t \leq p .
$$

Since $\mathcal{P}^{p} u_{p-1} \notin J$ by (3.4), we have

$$
r_{p} U_{p-1} \equiv a U_{p-1}^{2} \quad \bmod D^{3} B P^{*}(B X)+\left(p, v_{1}, v_{2}, \ldots\right)
$$

for some $a \in \mathbb{Z}_{(p)}$ with $a \neq \equiv \bmod p$, where $\mathbb{Z}_{(p)}$ is the ring of $p$-local integers.

Now we can put

$$
\begin{aligned}
r_{1} U_{p-1} \equiv & b_{1} U_{1} U_{p-1}+\sum_{k=3}^{(p+1) / 2} b_{k} U_{k} U_{p+1-k} \\
+ & v_{1}\left(c_{2} U_{2} U_{p-1}+\sum_{k=4}^{(p+1) / 2} c_{k} U_{k} U_{p+2-k}\right) \\
& \bmod D^{3} B P^{*}(B X)+\left(p, v_{1}, v_{2}, \ldots\right)^{2}
\end{aligned}
$$


with $b_{k}, c_{k} \in \mathbb{Z}_{(p)}$. Here, $r_{p-1} r_{1} \equiv p r_{p} \bmod \left(v_{1}, v_{2}, \ldots\right)$, and $r_{i} U_{p-i} \in$ $D^{2} B P^{*}(B X)+\left(p, v_{1}, \ldots\right)$ by (3.3). Thus

$$
\begin{aligned}
& p r_{p} U_{p-1} \equiv r_{p-1} r_{1} U_{p-1} \\
& \equiv b_{1}\left(r_{p-1} U_{1}\right) U_{p-1}+\sum_{k=3}^{(p+1 / 2)} b_{k}\left(r_{p-k} U_{k}\right)\left(r_{k-1} U_{p+1-k}\right) \\
&+p\left(c_{2}\left(r_{p-2} U_{2}\right) U_{p-1}+\sum_{k=4}^{(p+1) / 2} c_{k}\left(r_{p-k} U_{k}\right)\left(r_{k-2} U_{p+2-k}\right)\right) \\
& \equiv p b_{1} d U_{p-1}^{2} \\
& \quad \bmod D^{3} B P^{*}(B X)+\left(p^{2}, v_{1}, v_{2}, \ldots\right),
\end{aligned}
$$

where $r_{p-1} U_{1} \equiv p d U_{p-1} \bmod D^{2} B P^{*}(B X)+\left(p^{2}, v_{1}, v_{2}, \ldots\right)$ with $d \in \mathbb{Z}_{(p)}$. Thus we have $b_{1} d \equiv a \not \equiv 0 \bmod p$ by (3.5). In particular, we have

$$
r_{p-1} U_{1} \not \equiv 0 \quad \bmod D^{2} B P^{*}(B X)+\left(p^{2}, v_{1}, v_{2}, \ldots\right) .
$$

Suppose that $\mathcal{P}^{p-3} u_{1} \in D^{2} H^{*}\left(B X ; \mathbb{F}_{p}\right)$. Then

$$
r_{p-3} U_{1} \equiv p e U_{p-2} \quad \bmod D^{2} B P^{*}(B X)+\left(p^{2}, v_{1}, v_{2}, \ldots\right)
$$

for some $e \in \mathbb{Z}_{(p)}$. Thus, we have

$$
\begin{aligned}
r_{p-1} U_{1} \equiv\left(\begin{array}{c}
p-1 \\
2
\end{array}\right)^{-1} r_{2} r_{p-3} U_{1} & \equiv \text { per } r_{2} U_{p-2} \equiv 0 \\
& \bmod D^{2} B P^{*}(B X)+\left(p^{2}, v_{1}, v_{2}, \ldots\right),
\end{aligned}
$$

which is a contradiction. Therefore, we have

$$
\mathcal{P}^{p-3} u_{1} \notin D^{2} H^{*}\left(B X ; \mathbb{F}_{p}\right),
$$

and we can choose generators $u_{i}$ for $2 \leq i \leq p-2$ such that

$$
u_{i}=\left(\begin{array}{c}
p-2 \\
i-1
\end{array}\right)^{-1} \mathcal{P}^{i-1} u_{1}
$$

Thus by putting $x_{i}=s\left(u_{i}\right)$, where $s$ is the cohomology suspension, we have (3.2), which completes the proof.

Next we consider the $p$-compact group $X(m, m ; n)$.

Proposition 3.3. For any positive integers $m$ and $n$ with $m \mid p-1$, the p-compact group $X(m, m ; n)$ satisfies $(\mathcal{P})$. 
Proof. According to Castellana [6, p.2817] there is a map $B c: B X(q, 1 ; n)$ $\rightarrow B U(n q)_{p}^{\wedge}$ such that if we put

$$
u_{j}=B c^{*}\left(c_{j q}\right) \quad(1 \leq j \leq n)
$$

then

$$
H^{*}\left(B X(m, m ; n) ; \mathbb{F}_{p}\right)=\mathbb{F}_{p}\left[u_{1}, \ldots, u_{n}\right]
$$

where $c_{j}$ is the $j$-th Chern class so that

$$
H^{*}\left(B U(n q)_{p}^{\wedge} ; \mathbb{F}_{p}\right)=\mathbb{F}_{p}\left[c_{1}, \ldots, c_{n q}\right]
$$

Then by putting $x_{i}=s\left(u_{i}\right)$ for the cohomology suspension $s$ we have $(\mathcal{P})$ for $X(m, m ; n)$ from the one for $U(n q)_{p}^{\wedge}([4$, Corollaire 11.4], [16]).

Now we show Theorem 1.1.

Proof of Theorem 1.1. Let $X$ be a simple $p$-compact group of type $\left(n_{1}, \ldots\right.$, $\left.n_{k}\right)$ other than $X(m, q ; n)$ with $q<m$.

If $n_{k}-n_{1}<p-1$, then $(\mathcal{P})$ clearly holds since $X$ is $p$-regular and there are no $i$ and $j$ with $n_{j}-n_{i}=t(p-1)$ for any $t>0$.

Suppose that $p-1 \leq n_{k}-n_{1}<2(p-1)$. Then, by checking the Clark Ewing list, we can see that $n_{i} \neq n_{j}$ for $i \neq j$.

Let $n_{j}-n_{i}=t(p-1)$ for some $t>0$. Then it is clear that $t=1$. Moreover, by checking the Clark-Ewing list, we can see that $n_{j} \not \equiv 0 \bmod p$ and $P H^{2 s n_{j}-2(p-1)-1}\left(X ; \mathbb{F}_{p}\right)=0$ for $2 \leq s \leq p$. Thus by Lemma 3.1, $\mathcal{P}^{1}: P H^{2 n_{i}-1}\left(X ; \mathbb{F}_{p}\right) \rightarrow P H^{2 n_{j}-1}\left(X ; \mathbb{F}_{p}\right)$ is an epimorphism. Since $n_{i}-1 \equiv$ $n_{j} \not \equiv 0 \bmod p$, we can choose generators $x_{i}$ and $x_{j}$ with

$$
\mathcal{P}^{1} x_{i}=\left(\begin{array}{c}
n_{i}-1 \\
1
\end{array}\right) x_{j}
$$

Thus $(\mathcal{P})$ holds.

Now we consider the remaining case $n_{k}-n_{1} \geq 2(p-1)$. The ClarkEwing list consists of thirty seven families of irreducible $p$-adic pseudoreflection groups, with two subfamilies $2 \mathrm{a}$ and $2 \mathrm{~b}$ in No. 2. It is proved that all nonmodular irreducible $p$-adic pseudoreflection groups have realizations ([7]). On the other hand, not all modular irreducible $p$-adic pseudoreflection groups have realizations. The non-realizable ones for an odd prime $p$ are of Nos. 28, 35, 36 for $p=3$, and 37 for $p=3,5$ ([22], [2], [18], [19]). The following is the table of 
all realizable irreducible $p$-adic pseudoreflection groups with $n_{k}-n_{1} \geq 2(p-1)$.

\begin{tabular}{|l|l|l|}
\hline No. & \multicolumn{1}{|c|}{ type } & \multicolumn{1}{c|}{ prime } \\
\hline 1 & $(2,3, \ldots, n+1)$ & $p \leq(n+1) / 2$ \\
\hline $2 \mathrm{a}$ & $(m, 2 m, \ldots, n m)$ & $p \leq(n-1) m / 2+1$ \\
\hline $2 \mathrm{~b}$ & $(2,6)$ & 3 \\
\hline 26 & $(6,12,18)$ & 7 \\
\hline 28 & $(2,6,8,12)$ & 5 \\
\hline 29 & $(4,8,12,20)$ & 5 \\
\hline 30 & $(2,12,20,30)$ & 11 \\
\hline 31 & $(8,12,20,24)$ & 5 \\
\hline 32 & $(12,18,24,30)$ & 7 \\
\hline 33 & $(4,6,10,12,18)$ & 7 \\
\hline 34 & $(6,12,18,24,30,42)$ & $7,13,19$ \\
\hline 35 & $(2,5,6,8,9,12)$ & 5 \\
\hline 36 & $(2,6,8,10,12,14,18)$ & 5,7 \\
\hline 37 & $(2,8,12,14,18,20,24,30)$ & $7,11,13$ \\
\hline
\end{tabular}

Now, the $p$-adic pseudoreflection groups of Nos. $1,2 \mathrm{~b}, 28,35,36$ and 37 are the Weyl groups of the Lie groups $S U(n+1), G_{2}, F_{4}, E_{6}, E_{7}$ and $E_{8}$, respectively. If $B X$ realizes the Weyl group of a Lie group $G$, then $H^{*}\left(X ; \mathbb{F}_{p}\right)$ is isomorphic to $H^{*}\left(G ; \mathbb{F}_{p}\right)$ as an algebra over the Steenrod algebra. Thus, for these cases, we have only to show $(\mathcal{P})$ for corresponding Lie groups, which are proved in [4, Corollaire 11.4] (see also [16]), [3], [12] and [15].

For No. 2a, we have already done in Propositions 3.3.

For Nos. 29 for $p=5$ and 34 for $p=7,(\mathcal{P})$ holds by Proposition 3.2. For the Nos. 30 for $p=11$ and 31 for $p=5$, we can apply Lemma 3.1 to show $(\mathcal{P})$. Note that for No. 31, we have

$$
\mathcal{P}^{2} x_{2}=2^{-1} \mathcal{P}^{1} \mathcal{P}^{1} x_{2}=0=\left(\begin{array}{c}
12-1 \\
2
\end{array}\right) x_{3} .
$$

For No. 34 for $p=19,(\mathcal{P})$ holds by Nishinobu [17, Theorem 1.2].

Now we consider No. 32 for $p=7$. Then by Lemma 3.1 generators $x_{i}$ are chosen to satisfy

$$
\mathcal{P}^{1} x_{2}=\left(\begin{array}{c}
18-1 \\
1
\end{array}\right) x_{3}, \quad \mathcal{P}^{1} x_{3}=\left(\begin{array}{c}
24-1 \\
1
\end{array}\right) x_{4} .
$$

Thus, we have only to show that

$$
\mathcal{P}^{1} x_{1} \neq 0
$$


Then by modifying $x_{1}$ we have

$$
\mathcal{P}^{1} x_{1}=\left(\begin{array}{c}
12-1 \\
1
\end{array}\right) x_{2} .
$$

Suppose contrarily that $\mathcal{P}^{1} x_{1}=0$. Then, in $H^{*}\left(B X ; \mathbb{F}_{7}\right)=\mathbb{F}_{7}\left[u_{1}, u_{2}, u_{3}, u_{4}\right]$ the generators $u_{i}$ can be chosen to satisfy

$$
\mathcal{P}^{1} u_{1}=0, \quad \mathcal{P}^{1} u_{2}=\left(\begin{array}{c}
18-1 \\
1
\end{array}\right) u_{3}, \quad \mathcal{P}^{1} u_{3}=\left(\begin{array}{c}
24-1 \\
1
\end{array}\right) u_{4} .
$$

We show that $\mathcal{P}^{1} u_{4}$ is not in the ideal $\left(u_{1}, u_{3}, u_{4}\right)$ generated by $u_{1}, u_{3}, u_{4}$. In fact, if $\mathcal{P}^{1} u_{4} \in\left(u_{1}, u_{3}, u_{4}\right)$, then $\mathcal{P}^{1}\left(H^{*}\left(B X ; \mathbb{F}_{7}\right)\right) \subset\left(u_{1}, u_{3}, u_{4}\right)$ since $\mathcal{P}^{1} u_{i} \in$ $\left(u_{1}, u_{3}, u_{4}\right)$ for any $i=1,2,3,4$. On the other hand, we have

$$
\mathcal{P}^{1}\left(\mathcal{P}^{17} u_{2}\right)=18 \mathcal{P}^{18} u_{2}=18 u_{2}^{7} \notin\left(u_{1}, u_{3}, u_{4}\right) .
$$

This is a contradiction, and so we have

$$
\mathcal{P}^{1} u_{4} \notin\left(u_{1}, u_{3}, u_{4}\right) .
$$

Now, consider the quotient algebra $H^{*}\left(B X ; \mathbb{F}_{7}\right) /\left(u_{1}\right) \cong \mathbb{F}_{7}\left[\bar{u}_{2}, \bar{u}_{3}, \bar{u}_{4}\right]$, where $\bar{u}_{i}$ is the coset of $u_{i}$. Note that this algebra does not necessarily admit the Steenrod action, but has the $\mathcal{P}^{1}$ action. Then, by the above argument we can put

$$
\mathcal{P}^{1} \bar{u}_{4}=a \bar{u}_{2}^{2}
$$

with $a \neq 0$. Then by iterating $\mathcal{P}^{1}$ we have

$$
\left(\mathcal{P}^{1}\right)^{7} \bar{u}_{4}=3 a^{3} \bar{u}_{2}^{4}+a^{2} \bar{u}_{2} \bar{u}_{3} \bar{u}_{4}+3 a^{2} \bar{u}_{3}^{3} \neq 0 .
$$

This is a contradiction since $\left(\mathcal{P}^{1}\right)^{7}=0$. Thus $\mathcal{P}^{1} u_{1} \neq 0$ and we have $\mathcal{P}^{1} x_{1} \neq 0$.

The remaining cases are of Nos. 26 and 33 for $p=7$, and 34 for $p=13$. The idea of the proof for these cases are essentially the same. First we consider No. 26.

By Lemma 3.1 we can choose generators $x_{i}$ such that

$$
\mathcal{P}^{1} x_{2}=\left(\begin{array}{c}
12-1 \\
1
\end{array}\right) x_{3} .
$$

Thus, we prove

$$
\mathcal{P}^{1} x_{1} \neq 0
$$

to show

$$
\mathcal{P}^{1} x_{1}=\left(\begin{array}{c}
6-1 \\
1
\end{array}\right) x_{2}
$$


for a suitable $x_{1}$.

Suppose contrarily that $\mathcal{P}^{1} x_{1}=0$. Then we can choose generators $u_{i}$ $(i=1,2,3)$ of $H^{*}\left(B X ; \mathbb{F}_{p}\right)$ such that the ideal $\left(u_{1}\right)$ of $H^{*}\left(B X ; \mathbb{F}_{p}\right)$ generated by $u_{1}$ is closed under the action of the Steenrod algebra for dimensional reasons. Thus $H^{*}\left(B X ; \mathbb{F}_{p}\right) /\left(u_{1}\right)$ is a polynomial algebra of the type $(12,18)$ on which the Steenrod algebra acts unstably. According to Adams and Wilkerson [1, Theorem 1.2], the type of any non-modular polynomial algebra on which the Steenrod algebra acts unstably must be a union of types in the Clark-Ewing list, which is not the case. This is a contradiction, and $\mathcal{P}^{1} u_{1}$ is indecomposable. Thus we have $\mathcal{P}^{1} x_{1} \neq 0$.

Next consider No. 33.

By Lemma 3.1 we can choose generators $x_{i}$ such that

$$
\mathcal{P}^{1} x_{1}=\left(\begin{array}{c}
4-1 \\
1
\end{array}\right) x_{3}, \quad \mathcal{P}^{1} x_{4}=\left(\begin{array}{c}
12-1 \\
1
\end{array}\right) x_{5} .
$$

Thus we have only to show that

$$
\mathcal{P}^{1} x_{2} \neq 0
$$

to show

$$
\mathcal{P}^{1} x_{2}=\left(\begin{array}{c}
6-1 \\
1
\end{array}\right) x_{4}
$$

for a suitable $x_{2}$.

Suppose contrarily that $\mathcal{P}^{1} x_{2}=0$. Then we can choose generators $u_{i}$ of $H^{*}\left(B X ; \mathbb{F}_{p}\right)$ such that the ideal $\left(u_{1}, u_{2}, u_{3}\right)$ of $H^{*}\left(B X ; \mathbb{F}_{p}\right)$ generated by $u_{1}, u_{2}, u_{3}$ is closed under the action of the operation $\mathcal{P}^{1}$. Moreover, for dimensional reasons, we have $\mathcal{P}^{7} u_{3} \in\left(u_{1}, u_{2}, u_{3}\right)$. Thus $\left(u_{1}, u_{2}, u_{3}\right)$ is closed under the action of the Steenrod algebra, and we have a polynomial algebra $H^{*}\left(B X ; \mathbb{F}_{p}\right) /\left(u_{1}, u_{2}, u_{3}\right)$ of the type $(12,18)$ on which the Steenrod algebra acts unstably. Thus, by the same reason as the case of No. 26, we have a contradiction, and we have $\mathcal{P}^{1} x_{2} \neq 0$.

Finally, we consider No. 34 .

By Lemma 3.1, we can choose generators $x_{i}$ such that

$$
\mathcal{P}^{1} x_{2}=\left(\begin{array}{c}
12-1 \\
1
\end{array}\right) x_{4}, \quad \mathcal{P}^{1} x_{3}=\left(\begin{array}{c}
18-1 \\
1
\end{array}\right) x_{5}, \quad \mathcal{P}^{1} x_{5}=\left(\begin{array}{c}
30-1 \\
1
\end{array}\right) x_{6} .
$$

Thus, we have only to show that

$$
\mathcal{P}^{1} x_{1} \neq 0
$$


to show

$$
\mathcal{P}^{1} x_{1}=\left(\begin{array}{c}
6-1 \\
1
\end{array}\right) x_{3}
$$

for a suitable $x_{1}$.

Suppose contrarily that $\mathcal{P}^{1} x_{1}=0$. Then we can choose generators $u_{i}$ of $H^{*}\left(B X ; \mathbb{F}_{p}\right)$ such that the ideal $\left(u_{1}\right)$ of $H^{*}\left(B X ; \mathbb{F}_{p}\right)$ generated by $u_{1}$ is closed under the action of the Steenrod algebra for dimensional reasons. Thus we have a polynomial algebra $H^{*}\left(B X ; \mathbb{F}_{p}\right) /\left(u_{1}\right)$ of type $(12,18,24,30,42)$ on which the Steenrod algebra acts unstably. Thus, by the same reason as the case of No. 26 , we have a contradiction, and we have $\mathcal{P}^{1} x_{1} \neq 0$. This completes the proof of the theorem.

\section{$\S 4$. Steenrod Connection}

In this section we list the complete Steenrod actions on the cohomology of simple simply connected $p$-torsion free $p$-compact groups for any odd prime $p$. In No. 2 a we only consider $X(m, m ; n)$. For $X(m, q ; n)$ with $q<m$, the Steenrod actions can be given from the following isomorphism of algebras over the Steenrod algebra:

$$
H^{*}\left(X(m, q ; n) ; \mathbb{F}_{p}\right) \cong H^{*}\left(X(m, m ; n-1) ; \mathbb{F}_{p}\right) \otimes H^{*}\left(S^{2 n q-1} ; \mathbb{F}_{p}\right)
$$

In the following, we only list non trivial actions, where $P H^{n}$ denotes the $n$-dimensional primitive module of $H^{*}\left(X ; \mathbb{F}_{p}\right)$.

(No.1 of type: $(2,3, \ldots, n+1))$ For any odd prime $p$ with $p \leq n$ :

$$
\mathcal{P}^{k}\left(P H^{2 t-1}\right)=P H^{2(t+k(p-1))-1} \text { for }\left(\begin{array}{c}
t-1 \\
k
\end{array}\right) \not \equiv 0 \bmod p,
$$

where $t \geq 2$ and $t+k(p-1) \leq n+1$.

(No.2a of type: $(m, 2 m, \ldots, n m))$ For any odd prime $p$ with $p \leq n$ :

$$
\mathcal{P}^{k}\left(P H^{2 t m-1}\right)=P H^{2(t m+k(p-1))-1} \quad \text { for }\left(\begin{array}{c}
t m-1 \\
k
\end{array}\right) \not \equiv 0 \bmod p
$$

where $t \geq 1$ and $t m+k(p-1) \leq n m$.

(No.2b of type: $(2, p+1)$ For any odd prime $p$ :

$$
\mathcal{P}^{1}\left(P H^{3}\right)=P H^{2 p+1} \text {. }
$$


(No.5 of type: $(6,12)) \quad$ For $p=7$ :

$$
\mathcal{P}^{1}\left(P H^{11}\right)=P H^{23} .
$$

(No.8 of type: $(8,12)$ ) For $p=5$ :

$$
\mathcal{P}^{1}\left(P H^{15}\right)=P H^{23} .
$$

(No.9 of type: $(8,24)$ ) For $p=17$ :

$$
\mathcal{P}^{1}\left(P H^{15}\right)=P H^{47} .
$$

(No.10 of type: $(12,24)) \quad$ For $p=13$ :

$$
\mathcal{P}^{1}\left(P H^{23}\right)=P H^{47} \text {. }
$$

(No.12 of type: $(6,8)$ ) For $p=3$ :

$$
\mathcal{P}^{1}\left(P H^{11}\right)=P H^{15} .
$$

(No.14 of type: $(6,24)) \quad$ For $p=19$ :

$$
\mathcal{P}^{1}\left(P H^{11}\right)=P H^{47} .
$$

(No.16 of type: $(20,30))$ For $p=11$ :

$$
\mathcal{P}^{1}\left(P H^{39}\right)=P H^{59} .
$$

(No.17 of type: $(20,60)) \quad$ For $p=41$ :

$$
\mathcal{P}^{1}\left(P H^{39}\right)=P H^{119} \text {. }
$$

(No.18 of type: $(30,60)) \quad$ For $p=31$ :

$$
\mathcal{P}^{1}\left(P H^{59}\right)=P H^{119} \text {. }
$$

(No.20 of type: $(12,30)) \quad$ For $p=19$ :

$$
\mathcal{P}^{1}\left(P H^{23}\right)=P H^{59} .
$$

(No.24 of type: $(4,6,14)$ ) For $p=11$ :

$$
\mathcal{P}^{1}\left(P H^{7}\right)=P H^{27} .
$$

(No.25 of type: $(6,9,12))$ For $p=7$ :

$$
\mathcal{P}^{1}\left(P H^{11}\right)=P H^{23} .
$$


(No.26 of type: $(6,12,18)) \quad$ For $p=7$ :

$$
\begin{gathered}
\mathcal{P}^{1}\left(P H^{11}\right)=P H^{23}, \\
\mathcal{P}^{2}\left(P H^{11}\right)=\mathcal{P}^{1}\left(P H^{23}\right)=P H^{35} .
\end{gathered}
$$

For $p=13$ :

$$
\mathcal{P}^{1}\left(P H^{11}\right)=P H^{35} .
$$

(No.27 of type: $(6,12,30))$ For $p=19$ :

$$
\mathcal{P}^{1}\left(P H^{23}\right)=P H^{59} \text {. }
$$

(No.28 of type: $(2,6,8,12))$ For $p=5$ :

$$
\begin{gathered}
\mathcal{P}^{1}\left(P H^{3}\right)=P H^{11}, \\
\mathcal{P}^{1}\left(P H^{15}\right)=P H^{23} .
\end{gathered}
$$

For $p=7$ :

$$
\begin{gathered}
\mathcal{P}^{1}\left(P H^{3}\right)=P H^{15}, \\
\mathcal{P}^{1}\left(P H^{11}\right)=P H^{23} .
\end{gathered}
$$

For $p=11$ :

$$
\mathcal{P}^{1}\left(P H^{3}\right)=P H^{23} .
$$

(No.29 of type: $(4,8,12,20)) \quad$ For $p=5$ :

$$
\begin{gathered}
\mathcal{P}^{1}\left(P H^{7}\right)=P H^{15}, \\
\mathcal{P}^{2}\left(P H^{7}\right)=\mathcal{P}^{1}\left(P H^{15}\right)=P H^{23} .
\end{gathered}
$$

For $p=13$ :

$$
\mathcal{P}^{1}\left(P H^{15}\right)=P H^{39}
$$

For $p=17$ :

$$
\mathcal{P}^{1}\left(P H^{7}\right)=P H^{39} .
$$

(No.30 of type: $(2,12,20,30)) \quad$ For $p=11$ :

$$
\begin{gathered}
\mathcal{P}^{1}\left(P H^{3}\right)=P H^{23}, \\
\mathcal{P}^{1}\left(P H^{39}\right)=P H^{59} .
\end{gathered}
$$

For $p=19$ :

$$
\begin{aligned}
\mathcal{P}^{1}\left(P H^{3}\right) & =P H^{39}, \\
\mathcal{P}^{1}\left(P H^{23}\right) & =P H^{59} .
\end{aligned}
$$


For $p=29$ :

$$
\mathcal{P}^{1}\left(P H^{3}\right)=P H^{59} .
$$

(No.31 of type: $(8,12,20,24))$ For $p=5$ :

$$
\begin{aligned}
& \mathcal{P}^{1}\left(P H^{15}\right)=P H^{23}, \\
& \mathcal{P}^{1}\left(P H^{39}\right)=P H^{47} .
\end{aligned}
$$

For $p=13$ :

$$
\begin{aligned}
& \mathcal{P}^{1}\left(P H^{15}\right)=P H^{39}, \\
& \mathcal{P}^{1}\left(P H^{23}\right)=P H^{47} .
\end{aligned}
$$

For $p=17$ :

$$
\mathcal{P}^{1}\left(P H^{15}\right)=P H^{47} .
$$

(No.32 of type: $(12,18,24,30))$ For $p=7$ :

$$
\begin{gathered}
\mathcal{P}^{1}\left(P H^{23}\right)=P H^{35}, \\
\mathcal{P}^{2}\left(P H^{23}\right)=\mathcal{P}^{1}\left(P H^{35}\right)=P H^{47}, \\
\mathcal{P}^{3}\left(P H^{23}\right)=\mathcal{P}^{2}\left(P H^{35}\right)=\mathcal{P}^{1}\left(P H^{47}\right)=P H^{59} .
\end{gathered}
$$

For $p=13$ :

$$
\begin{aligned}
& \mathcal{P}^{1}\left(P H^{23}\right)=P H^{47}, \\
& \mathcal{P}^{1}\left(P H^{35}\right)=P H^{59} .
\end{aligned}
$$

For $p=19$ :

$$
\mathcal{P}^{1}\left(P H^{23}\right)=P H^{59} .
$$

(No.33 of type: $(4,6,10,12,18))$ For $p=7$ :

$$
\begin{gathered}
\mathcal{P}^{1}\left(P H^{7}\right)=P H^{19}, \\
\mathcal{P}^{1}\left(P H^{11}\right)=P H^{23}, \\
\mathcal{P}^{2}\left(P H^{11}\right)=\mathcal{P}^{1}\left(P H^{23}\right)=P H^{35} .
\end{gathered}
$$

For $p=13$ :

$$
\mathcal{P}^{1}\left(P H^{11}\right)=P H^{35} .
$$

(No.34 of type: $(6,12,18,24,30,42)) \quad$ For $p=7$ :

$$
\begin{gathered}
\mathcal{P}^{1}\left(P H^{11}\right)=P H^{23}, \\
\mathcal{P}^{2}\left(P H^{11}\right)=\mathcal{P}^{1}\left(P H^{23}\right)=P H^{35}, \\
\mathcal{P}^{3}\left(P H^{11}\right)=\mathcal{P}^{2}\left(P H^{23}\right)=\mathcal{P}^{1}\left(P H^{35}\right)=P H^{47}, \\
\mathcal{P}^{4}\left(P H^{11}\right)=\mathcal{P}^{3}\left(P H^{23}\right)=\mathcal{P}^{2}\left(P H^{35}\right)=\mathcal{P}^{1}\left(P H^{47}\right)=P H^{59} .
\end{gathered}
$$


For $p=13$ :

$$
\begin{gathered}
\mathcal{P}^{1}\left(P H^{11}\right)=P H^{35}, \\
\mathcal{P}^{1}\left(P H^{23}\right)=P H^{47}, \\
\mathcal{P}^{2}\left(P H^{11}\right)=\mathcal{P}^{1}\left(P H^{35}\right)=P H^{59}, \\
\mathcal{P}^{3}\left(P H^{11}\right)=\mathcal{P}^{2}\left(P H^{35}\right)=\mathcal{P}^{1}\left(P H^{59}\right)=P H^{83} .
\end{gathered}
$$

For $p=19$ :

$$
\begin{gathered}
\mathcal{P}^{1}\left(P H^{11}\right)=P H^{47}, \\
\mathcal{P}^{1}\left(P H^{23}\right)=P H^{59}, \\
\mathcal{P}^{2}\left(P H^{11}\right)=\mathcal{P}^{1}\left(P H^{47}\right)=P H^{83} .
\end{gathered}
$$

For $p=31$ :

$$
\mathcal{P}^{1}\left(P H^{23}\right)=P H^{83}
$$

For $p=37$ :

$$
\mathcal{P}^{1}\left(P H^{11}\right)=P H^{83} .
$$

(No.35 of type: $(2,5,6,8,9,12))$ For $p=5$ :

$$
\begin{gathered}
\mathcal{P}^{1}\left(P H^{3}\right)=P H^{11}, \\
\mathcal{P}^{1}\left(P H^{9}\right)=P H^{17}, \\
\mathcal{P}^{1}\left(P H^{15}\right)=P H^{23} .
\end{gathered}
$$

For $p=7$ :

$$
\begin{gathered}
\mathcal{P}^{1}\left(P H^{3}\right)=P H^{15}, \\
\mathcal{P}^{1}\left(P H^{11}\right)=P H^{23} .
\end{gathered}
$$

For $p=11$ :

$$
\mathcal{P}^{1}\left(P H^{3}\right)=P H^{23} .
$$

(No.36 of type: $(2,6,8,10,12,14,18))$ For $p=5$ :

$$
\begin{gathered}
\mathcal{P}^{1}\left(P H^{3}\right)=P H^{11}, \\
\mathcal{P}^{1}\left(P H^{15}\right)=P H^{23}, \\
\mathcal{P}^{1}\left(P H^{19}\right)=P H^{27}, \\
\mathcal{P}^{2}\left(P H^{19}\right)=\mathcal{P}^{1}\left(P H^{27}\right)=P H^{35} .
\end{gathered}
$$


For $p=7$ :

$$
\begin{gathered}
\mathcal{P}^{1}\left(P H^{3}\right)=P H^{15}, \\
\mathcal{P}^{1}\left(P H^{11}\right)=P H^{23}, \\
\mathcal{P}^{2}\left(P H^{11}\right)=\mathcal{P}^{1}\left(P H^{23}\right)=P H^{35} .
\end{gathered}
$$

For $p=11$ :

$$
\begin{gathered}
\mathcal{P}^{1}\left(P H^{3}\right)=P H^{23}, \\
\mathcal{P}^{1}\left(P H^{15}\right)=P H^{35} .
\end{gathered}
$$

For $p=13$ :

$$
\begin{aligned}
& \mathcal{P}^{1}\left(P H^{3}\right)=P H^{27}, \\
& \mathcal{P}^{1}\left(P H^{11}\right)=P H^{35} .
\end{aligned}
$$

For $p=17$ :

$$
\mathcal{P}^{1}\left(P H^{3}\right)=P H^{35} .
$$

(No.37 of type: $(2,8,12,14,18,20,24,30))$ For $p=7$ :

$$
\begin{gathered}
\mathcal{P}^{1}\left(P H^{3}\right)=P H^{15}, \\
\mathcal{P}^{1}\left(P H^{23}\right)=P H^{35}, \\
\mathcal{P}^{1}\left(P H^{27}\right)=P H^{39}, \\
\mathcal{P}^{2}\left(P H^{23}\right)=\mathcal{P}^{1}\left(P H^{35}\right)=P H^{47}, \\
\mathcal{P}^{3}\left(P H^{23}\right)=\mathcal{P}^{2}\left(P H^{35}\right)=\mathcal{P}^{1}\left(P H^{47}\right)=P H^{59} .
\end{gathered}
$$

For $p=11$ :

$$
\begin{aligned}
\mathcal{P}^{1}\left(P H^{3}\right) & =P H^{23}, \\
\mathcal{P}^{1}\left(P H^{15}\right) & =P H^{35}, \\
\mathcal{P}^{1}\left(P H^{27}\right) & =P H^{47}, \\
\mathcal{P}^{1}\left(P H^{39}\right) & =P H^{59} .
\end{aligned}
$$

For $p=13$ :

$$
\begin{aligned}
\mathcal{P}^{1}\left(P H^{3}\right) & =P H^{27}, \\
\mathcal{P}^{1}\left(P H^{15}\right) & =P H^{39}, \\
\mathcal{P}^{1}\left(P H^{23}\right) & =P H^{47}, \\
\mathcal{P}^{1}\left(P H^{35}\right) & =P H^{59} .
\end{aligned}
$$


For $p=17$ :

$$
\begin{aligned}
\mathcal{P}^{1}\left(P H^{3}\right) & =P H^{35}, \\
\mathcal{P}^{1}\left(P H^{15}\right) & =P H^{47}, \\
\mathcal{P}^{1}\left(P H^{27}\right) & =P H^{59} .
\end{aligned}
$$

For $p=19$ :

$$
\begin{gathered}
\mathcal{P}^{1}\left(P H^{3}\right)=P H^{39}, \\
\mathcal{P}^{1}\left(P H^{23}\right)=P H^{59} .
\end{gathered}
$$

For $p=23$ :

$$
\begin{gathered}
\mathcal{P}^{1}\left(P H^{3}\right)=P H^{47}, \\
\mathcal{P}^{1}\left(P H^{15}\right)=P H^{59} .
\end{gathered}
$$

For $p=29$ :

$$
\mathcal{P}^{1}\left(P H^{3}\right)=P H^{59}
$$

\section{References}

[1] J. F. Adams and C. W. Wilkerson, Finite $H$-spaces and algebras over the Steenrod algebra, Ann. of Math. (2) 111 (1980), no. 1, 95-143.

[2] J. Aguadé, Constructing modular classifying spaces, Israel J. Math. 66 (1989), no. 1-3, $23-40$.

[3] A. Borel, Sur la cohomologie des espaces fibrés principaux et des espaces homogènes de groupes de Lie compacts, Ann. of Math. (2) 57 (1953), 115-207.

[4] A. Borel and J.-P. Serre, Groupes de Lie et puissances réduites de Steenrod, Amer. J. Math. 75 (1953), 409-448.

[5] N. Castellana, Quillen Grassmannians as non-modular homotopy fixed points, Math. Z. 248 (2004), no. 3, 477-493.

[6] On the $p$-compact groups corresponding to the $p$-adic reflection groups $G(q, r, n)$, Trans. Amer. Math. Soc. 358 (2006), no. 7, 2799-2819 (electronic).

7] A. Clark and J. Ewing, The realization of polynomial algebras as cohomology rings, Pacific J. Math. 50 (1974), 425-434.

[8] W. G. Dwyer, H. R. Miller and C. W. Wilkerson, Homotopical uniqueness of classifying spaces, Topology 31 (1992), no. 1, 29-45.

[9] W. G. Dwyer and C. W. Wilkerson, Homotopy fixed-point methods for Lie groups and finite loop spaces, Ann. of Math. (2) 139 (1994), no. 2, 395-442.

[10] Y. Hemmi, On finite $H$-spaces given by sphere extensions of classical groups, Hiroshima Math. J. 14 (1984), no. 3, 451-470.

[11] Homotopy associative finite $H$-spaces and the mod 3 reduced power operations, Publ. Res. Inst. Math. Sci. 23 (1987), no. 6, 1071-1084.

[12] P. G. Kumpel, Jr., Lie groups and products of spheres, Proc. Amer. Math. Soc. 16 (1965), 1350-1356.

[13] J. P. Lin, Torsion in $H$-spaces. II, Ann. Math. (2) 107 (1978), no. 1, 41-88. 
[14] J. W. Milnor and J. D. Stasheff, Characteristic classes, Ann. of Math. Stud., 76, Princeton Univ. Press, Princeton, N.J., 1974.

[15] M. Mimura and H. Toda, Cohomology operations and homotopy of compact Lie groups. I, Topology 9 (1970), 317-336.

[16] S. Mukohda and S. Sawaki, On the $b_{p}^{k, j}$ coefficient of a certain symmetric function, J. Fac. Sci. Niigata Univ. Ser. I. 1 (1954), no. 2, 6 pp.

[17] H. Nishinobu, On the action of the Steenrod algebra on the cohomology of a certain p-compact group, Kochi J. Math. 2 (2007), 125-153.

[18] D. Notbohm, For which pseudo-reflection groups are the $p$-adic polynomial invariants again a polynomial algebra?, J. Algebra 214 (1999), no. 2, 553-570.

[19] _ Erratum: "For which pseudo-reflection groups are the $p$-adic polynomial invariants again a polynomial algebra?" J. Algebra 218 (1999), no. 1, 286-287.

[20] G. C. Shephard and J. A. Todd, Finite unitary reflection groups, Canadian J. Math. 6 (1954), 274-304.

[21] E. Thomas, Steenrod squares and $H$-spaces. II, Ann. of Math. (2) 81 (1965), 473-495

[22] A. Zabrodsky, On the realization of invariant subgroups of $\pi_{*}(X)$, Trans. Amer. Math. Soc. 285 (1984), no. 2, 467-496. 